\title{
Estudio retrospectivo que evalúa la carga de la varicela en México en menores de 1-14 años tratados en 10 sitios
}

\section{Retrospective Study Assessing the Burden of Varicella in México in Children 1-14 Years of Age Treated Across 10 Sites}

\author{
Mirella Vázquez-Rivera, ${ }_{1}^{1}$ Carlos Pérez Bolde-Villarreal, ${ }^{2}$ Homero Monsanto, ${ }^{3}$ Emmanouil Rampakakis,${ }^{4}$ Alexandra \\ Altland, ${ }^{5}$ Lara J Wolfson ${ }^{5}$ \\ *Grupo de Estudio MARVEL México, Erika González-Flores, ${ }^{1}$ Claudia Jiménez-Vargas, ${ }^{2}$ Miguel Ángel Martínez- \\ Romero, ${ }^{3}$ Alma Pérez-Ríos, ${ }^{4}$ Roselia Ramírez-Rivera, ${ }^{5}$ Eneida Sánchez-Medina, ${ }^{6}$ Jorge Alejandro Vázquez- \\ Narváez ${ }^{7}$, Ana Luisa Ortiz-Rivera, ${ }^{8}$ Francisco Moreno-Ramos ${ }^{9}$
}

\section{Resumen}

OBJETIVO: Evaluar la carga clínica y económica de la varicela en pacientes mexicanos de 1-14 años.

MÉTODOS: Estudio retrospectivo, multicéntrico, de expedientes clínicos de pacientes de 1-14 años con diagnóstico de varicela primaria atendidos del 2011 al 2016 en 10 sitios. Los costos directos e indirectos individuales (dólares americanos al tipo de cambio promedio de 2017) se calcularon a partir del uso de recursos de atención médica, los gastos pagados por los padres o tutores del paciente y la pérdida de trabajo de los cuidadores. Los costos sociales anuales se estimaron para un escenario base multiplicando los costos por paciente por la incidencia promedio de la varicela según el Sistema Único de Información para la Vigilancia Epidemiológica (SUIVE).

RESULTADOS: Se incluyeron los expedientes clínicos de 152 pacientes con varicela: 75 ambulatorios y 77 hospitalizados. Estos últimos reportaron tasas de complicaciones más altas $(84.4$ vs $6.7 \%)$, requirieron más medicamentos recetados y de venta libre $(94.8 \mathrm{vs}$ $80.0 \% ; 71.4$ vs $80.0 \%$, respectivamente), análisis y procedimientos (87.0 vs $5.3 \%$ ). Los costos directos e indirectos se calcularon en 198.3 y 42.3 dólares por paciente ambulatorio, y en 5611 y 175.2 dólares por paciente hospitalizado, respectivamente. CONCLUSIÓN: Existe una carga clínica y económica considerable entre los menores de edad con varicela primaria en México. Estos resultados destacan la necesidad de implementar un programa de vacunación infantil contra la varicela.

PALABRAS CLAVE: Niño; varicela; vacunación; México; carga económica de la enfermedad; uso de recursos de atención médica

\section{Abstract}

OBJECTIVE: Evaluate the clinical and economic burden of Varicella in Mexican patients age 1 to 14 years old.

METHODS: Multicenter, retrospective chart review in Mexican patients aged 1-14 years diagnosed with primary varicella during 2011-2016 treated across 10 sites. Individual direct and indirect costs (2017 USD) were calculated using patient-reported healthcare resource utilization attributable to varicella, and caregiver work loss. Annual societal costs were estimated for a base scenario by multiplying per-patient costs with mean 2011-2016 Mexican epidemiological surveillance system (SUIVE) varicella incidence, inflated for the estimated proportion of public healthcare centers not regularly reporting to SUIVE.

RESULTS: Charts of 152 varicella patients were included: 75 outpatients and 77 inpatients. Inpatients reported higher complication rates (84.4 vs 6.7\%), prescription and over-the

\author{
Instituto Nacional de Pediatría, Ciudad \\ de México. \\ ${ }^{2}$ MSD, México. \\ ${ }^{3}$ Merck Sharp \& Dohme IA LLC, Carolina, \\ Puerto Rico. \\ ${ }^{4}$ JSS Medical Research, Montreal, Que- \\ bec, Canadá. \\ ${ }^{5}$ Merck \& Co., Inc., Kenilworth, NJ USA. \\ *Grupo de estudio MARVEL México \\ ${ }^{1}$ Hospital del Niño y el Adolescente \\ Morelense, Emiliano Zapata, Morelos, \\ México. \\ 2 Hospital Materno Infantil ISSEMyM \\ Toluca, Estado de México. \\ ${ }^{3}$ Hospital de Especialidades del ISSSTEP, \\ Puebla de los Ángeles, Puebla, México. \\ ${ }^{4}$ Centro de Investigación Farmacéutica \\ Especializada, Guadalajara, Jalisco, \\ México. \\ ${ }^{5}$ Hospital de Especialidades del Niño y \\ la Mujer, SESEQ, Santiago de Querétaro, \\ Querétaro, México. \\ ${ }^{6}$ Hospital Infantil de Tlaxcala, Tlaxcala de \\ Xicoténcatl, Tlaxcala, México. \\ 7 Hospital Infantil de Morelia Eva Sámano, \\ Morelia, Michoacán de Ocampo, México. \\ ${ }^{8}$ Instituto Metropolitano de Investigación \\ Clínica SC. \\ ${ }^{9}$ Consultorio privado, Ciudad de México.
}

Recibido: 17 de mayo 2018

Aceptado: 10 de septiembre 2018

Correspondencia

Lara J. Wolfson

lara.wolfson@merck.com

Este artículo debe citarse como

Vázquez-Rivera $\mathrm{M}$, Pérez Bolde-Villarreal $C$, Monsanto $H$, Rampakakis $E$, Altland A, Wolfson $\amalg$, Grupo de Estudio MARVEL México. Estudio retrospectivo que evalúa la carga de la varicela en México en menores de 1-14 años tratados en 10 sitios. Acta Pediatr Mex. 2018;39(6):334-348. 


\begin{abstract}
counter medication use (94.8 vs $80.0 \% ; 71.4$ vs $80.0 \%$, respectively), tests/procedures ( 87.0 vs $5.3 \%$ ), and allied health professional consultations (61.0 vs $12.0 \%$ ). Direct and indirect costs were calculated at 198.3 and 42.3 dólares per outpatient, and 5,611.0 and 175.2 USD per inpatient, respectively.

CONCLUSION: Substantial clinical and economic burden was observed among children with primary varicella in Mexico. These results highlight the need for implementation of a childhood varicella vaccination program.

KEYWORDS: Child; Varicella; Vaccination; Mexico; Economic Burden of Disease; Healthcare Resource Utilization.
\end{abstract}

\section{INTRODUCCIÓN}

La varicela, también conocida como viruela loca, es una enfermedad infecciosa frecuente en niños, causada por el virus varicela-zóster, que es sumamente contagioso y puede trasmitirse por contacto directo o inhalación. ${ }^{1}$ Luego de la exposición al virus varicela-zóster y de un periodo de incubación de 10 días a 3 semanas puede aparecer un ligero pródromo de febrícula, malestar general, pérdida de apetito, dolor de cabeza y malestar abdominal, ${ }^{2}$ seguida de la erupción vesicular prurítica distintiva, 1 a 2 días más tarde. ${ }^{2}$ En ocasiones, las manifestaciones son más graves: síntomas neurológicos, gastrointestinales, respiratorios, complicaciones cutáneas y de tejidos blandos, y neumonía. .-5 $^{3-5}$

En México, una publicación reciente estimó que la incidencia anual promedio de varicela, entre 2000-2013, fue de aproximadamente 300,000 casos, la mayoría (57\%) en menores de 9 años. ${ }^{6}$ En el país, los menores con síntomas de varicela suelen ser atendidos en instalaciones de atención primaria y existe un flujograma de tratamiento que indica cómo debe administrarse tal atención. ${ }^{7}$ Si bien en la mayoría de los casos la varicela es una enfermedad de alivio espontáneo, después de la confirmación del diagnóstico los pacientes pueden tratarse, en caso de complicaciones, con antipiréticos orales, antihistamínicos, antipruríticos tópicos y antivirales orales. Si los síntomas son alarmantes se recomienda el traslado de los pacientes a los servicios de urgencias pediátricas de hospitales de segundo o tercer nivel de atención.

En México, para la prevención de la varicela se dispone de vacunas vivas atenuadas contra el virus varicela-zóster. Su aplicación está restringida a consultorios médicos privados y a menores de edad que reciben trasplante de médula ósea, aunque también se recomiendan para pacientes pediátricos en la guía de práctica clínica de la Secretaría de Salud. ${ }^{7-10}$ A pesar de no disponerse de registros oficiales de la cobertura de vacunación se estima que en México, antes de 2012, 1-2\% de los menores de edad se vacunaron contra el virus varicela-zóster, sobre todo en grandes ciudades y con pediatras privados. ${ }^{11}$ Empero, en México se cuenta con pocos estudios epidemiológicos que cuantifiquen la carga de la enfermedad asociada con la varicela y el posible efecto de la introducción de un programa de vacunación infantil contra la varicela.

El objetivo de este estudio fue: examinar la incidencia de complicaciones, uso de recursos de atención médica y los costos directos e indirectos asociados entre los pacientes pediátricos hospitalizados y ambulatorios con diagnóstico de varicela en México entre 2011 y 2016. En la 
bibliografía hay poca información referente a la carga social y económica de la varicela en México. Los datos pueden ayudar a poblar modelos económicos de salud para evaluar la rentabilidad de un programa de vacunación universal contra la varicela con financiamiento público.

\section{MATERIALES Y MÉTODOS}

Ensayo retrospectivo, multicéntrico, de expedientes clínicos, efectuado en 10 centros mexicanos (6 hospitales públicos y 4 clínicas privadas), como parte de un estudio internacional que incluyó a: Argentina, Jordania, México, Perú, Polonia y Hungría, ${ }^{12}$ y fue conducido conforme a los lineamientos de las buenas prácticas de farmacoepidemiología. El estudio fue aprobado por los comités de ética de las instituciones participantes: Instituto Nacional de Pediatría, Hospital Médica Sur, Hospital de Especialidades del Niño y la Mujer, Hospital del Niño y el Adolescente Morelense, y el Centro de Investigación Farmacéutica Especializada. No se exigió el consentimiento del paciente debido a la naturaleza retrospectiva del estudio y la recopilación anónima de datos.

Los casos incluidos se diagnosticaron en un lapso de cinco años que precedió al inicio del estudio y estuvieron sujetos al seguimiento posterior de la fecha del diagnóstico inicial de varicela, hasta la fecha de la desaparición o último día de contacto.

Se planificó incluir 150 casos, en proporción aproximada 1:1 entre pacientes ambulatorios y hospitalizados, como una muestra de conveniencia. A fin de ser elegibles para el estudio, los pacientes debían tener entre 1 y 14 años al momento del diagnóstico inicial de varicela. Los pacientes ambulatorios podían haber visitado el consultorio del médico (médico de cabecera, general, pediatra o infectólogo), una clínica ambulatoria o departamento ambulatorio de hospital, o una sala de urgencias sin necesidad de hospitalización. Los pacientes hospitalizados se definieron como menores de edad admitidos en un hospital debido a la varicela y sus complicaciones. Los pacientes examinados, inicialmente en circunstancias ambulatorias, por ejemplo, en una clínica o sala de urgencias, y que fueron hospitalizados posteriormente, se clasificaron como pacientes hospitalizados. Los casos de vacunación previa contra la varicela, los casos posteriores de varicela, y los pacientes con diagnóstico de herpes zóster, se excluyeron del estudio.

\section{Medición de los resultados}

Todos los resultados evaluados se extrajeron de los expedientes clínicos de los pacientes. Las complicaciones clínicas asociadas con la varicela fueron: infecciones de piel y tejidos blandos, meningitis, encefalitis, neumonía, septicemia, osteomielitis aguda, artritis séptica, cerebelitis, queratoconjuntivitis, hepatitis, nefritis, convulsiones febriles, deshidratación, dolor intenso, trastorno de la coagulación, y otros, según corresponda. En lo que respecta a uso de recursos de atención médica, se evaluaron los siguientes recursos: número de visitas ambulatorias y por tipo (consultorio médico, sala de urgencias, clínica ambulatoria de hospital); número y duración de las hospitalizaciones y visitas a cuidados intensivos de pacientes hospitalizados; medicamentos recetados y medicamentos de venta libre; cantidad de análisis y/o procedimientos efectuados; y cantidad de contactos de atención no médica. Con base en el uso de recursos de atención médica y las pérdidas de ingresos de los cuidadores, se estimaron los costos directos e indirectos asociados con la varicela por caso.

\section{Métodos estadísticos}

Todos los pacientes inscritos en el estudio se incluyeron en el análisis, que se estratificó por condiciones de salud de paciente hospitalizado 
vs paciente ambulatorio. Para conseguir los objetivos del estudio se produjeron estadísticas descriptivas; en el caso de las variables continuas se obtuvieron: promedio, desviación estándar e intervalos de confianza de $95 \%$ de los promedios, en tanto que para las variables categóricas se produjeron distribuciones de frecuencia. No hubo imputaciones de datos faltantes.

Para determinar los costos directos, cantidad de recursos que cada paciente utilizó, se multiplicó por el costo unitario de cada recurso en 2017, según lo publicado por el Instituto Mexicano del Seguro Social. ${ }^{13,14}$ Los costos indirectos se evaluaron estimando las pérdidas de ingresos de los cuidadores, de acuerdo con las estadísticas de ingresos promedio nacionales reportadas por el Instituto Mexicano del Seguro Social..$^{15}$ En el caso de los cuidadores de pacientes ambulatorios, se estimaron 2.5 días de trabajo perdidos con base en los estudios anteriores ${ }^{16,17}$ y en el caso de los pacientes hospitalizados, se calculó de manera independiente la duración (en días) de la hospitalización y la estancia en cuidados intensivos. No se aplicó ninguna tasa de inflación al calcular los costos por paciente con el tiempo.

El costo anual estimado de la varicela por cada año del estudio se calculó con base en la cantidad anual promedio de casos de varicela pediátrica ( $\leq 15$ años de edad), reportada al Sistema Único de Información para la Vigilancia Epidemiológica (SUIVE), el sistema mexicano de vigilancia epidemiológica, en el periodo 2011$2016 .{ }^{18-23}$ Se estableció un escenario básico en el que los casos de varicela reportados realmente al SUIVE se inflaron para compensar $4.2 \%$ de los centros de salud locales que no recopilan datos para el SUIVE, y $16 \%$ de los que no envían datos en forma semanal. ${ }^{24}$ El límite inferior del análisis de sensibilidad se basó en la cantidad promedio anual bruta de casos de varicela pediátrica reportados al SUIVE cada año, mientras que el límite superior se aplicó al escenario básico, una tasa de inflación de 25\% para reflejar la proporción estimada de pacientes del sector público que utilizan servicios privados. ${ }^{25}$

La cantidad de pacientes hospitalizados vs pacientes ambulatorios se definió con base en los datos de América Latina acerca de la proporción de solicitantes de atención médica que son hospitalizados debido a la varicela, que se estima en $5.78 \%{ }^{26}$ Los costos anuales (directos e indirectos) correspondientes se calcularon conforme a la cotización del peso mexicano respecto del dólar americano en 2017: 1 peso = 0.051 dólares. ${ }^{27}$ El análisis estadístico se efectuó con el Programa SAS ${ }^{\circledR}$, versión 9.4 (SAS Institute Inc., Cary, NC, Estados Unidos).

\section{RESULTADOS}

Se incluyeron 152 pacientes con varicela primaria elegibles durante 2011-2016; de estos 75 fueron ambulatorios y 77 hospitalizados. En el Cuadro 1 se presentan las características generales de la población por situación de salud de paciente hospitalizado o ambulatorio. La edad promedio \pm DE fue de $3.0 \pm 3.2$ años entre los pacientes ambulatorios, y de $2.6 \pm 3.5$ años entre los hospitalizados. La mayoría de los pacientes ambulatorios fue de sexo femenino ( $n=44 / 75 ; 58.7 \%$ ), pero se observó una distribución equiparable de los sexos entre los pacientes hospitalizados. En lo que respecta a la gravedad de la enfermedad, la proporción de pacientes hospitalizados que reportaron lesiones fue más alta, lo que indicó enfermedad más grave: 250-500 lesiones (pacientes ambulatorios: $5.3 \%$ vs hospitalizados: $14.3 \%$ ) y más de 500 lesiones (pacientes ambulatorios: $0.0 \%$ vs hospitalizados: $1.3 \%$ ).

La incidencia de complicaciones de la varicela fue más alta en los pacientes hospitalizados, pues $84.4 \%(n=65 / 77)$ experimentaron, al menos, una complicación en comparación con 
Cuadro 1. Características del paciente y la enfermedad al momento del diagnóstico de varicela

\begin{tabular}{|c|c|c|}
\hline & $\begin{array}{c}\text { Pacientes } \\
\text { ambulatorios } \\
(\mathbf{n}=75)\end{array}$ & $\begin{array}{c}\text { Pacientes } \\
\text { hospitalizados } \\
(\mathbf{n}=\mathbf{7 7})\end{array}$ \\
\hline \multicolumn{3}{|l|}{$\begin{array}{l}\text { Características del } \\
\text { paciente }\end{array}$} \\
\hline $\begin{array}{l}\text { Edad, años, promedio } \\
\pm \mathrm{DE}\end{array}$ & $3.0 \pm 3.2$ & $2.6 \pm 3.5$ \\
\hline \multicolumn{3}{|l|}{ Sexo, n (\%) } \\
\hline Masculino & 31 (41.3\%) & 39 (50.6 \%) \\
\hline Femenino & $44(58.7 \%)$ & $38(49.4 \%)$ \\
\hline \multicolumn{3}{|l|}{$\begin{array}{l}\text { Lugar de residencia, } \\
\text { n (\%) }\end{array}$} \\
\hline Urbano & $62(82.7 \%)$ & $61(79.2 \%)$ \\
\hline Rural & 13 (17.3 \%) & $16(20.8 \%)$ \\
\hline \multicolumn{3}{|l|}{$\begin{array}{l}\text { Año calendario del } \\
\text { diagnóstico, n (\%) }\end{array}$} \\
\hline 2011 & $1(1.3 \%)$ & $4(5.2 \%)$ \\
\hline 2012 & $5(6.7 \%)$ & $10(13.0 \%)$ \\
\hline 2013 & $5(6.7 \%)$ & $8(10.4 \%)$ \\
\hline 2014 & $19(25.3 \%)$ & $16(20.8 \%)$ \\
\hline 2015 & $31(41.3 \%)$ & $20(26.0 \%)$ \\
\hline 2016 & $14(18.7 \%)$ & $19(24.7 \%)$ \\
\hline $\begin{array}{l}\text { Pacientes inmunocom- } \\
\text { prometidos }^{\dagger}, \mathbf{n}(\%)\end{array}$ & $1(1.3 \%)$ & $5(6.5 \%)$ \\
\hline \multicolumn{3}{|l|}{$\begin{array}{l}\text { Características de la } \\
\text { enfermedad }\end{array}$} \\
\hline \multicolumn{3}{|l|}{$\begin{array}{l}\text { Cantidad máxima de le- } \\
\text { siones cutáneas durante } \\
\text { la erupción, } \mathrm{n}(\%)\end{array}$} \\
\hline$<50$ & $27(36.0 \%)$ & $20(26.0 \%)$ \\
\hline $50-249$ & $44(58.7 \%)$ & 45 (58.4 \%) \\
\hline $250-500$ & $4(5.3 \%)$ & $11(14.3 \%)$ \\
\hline$>500$ & $0(0.0 \%)$ & $1(1.3 \%)$ \\
\hline
\end{tabular}

$\mathrm{DE}=$ desviación estándar.

${ }^{+}$Los pacientes se consideraron inmunocomprometidos si tenían, al menos, una de las siguientes condiciones: VIH o SIDA, inmunodeficiencia congénita, tratamiento con corticosteroides sistémicos, o cualquier otro padecimiento inmunodebilitante mencionado en sus antecedentes médicos.

$6.7 \%(n=5 / 75)$ de los pacientes ambulatorios (Figura 1a). Entre los pacientes ambulatorios, las complicaciones más frecuentes fueron las infecciones de piel y tejidos blandos $(37.5 \%$ de las complicaciones; $n=3 / 8$ ). Por lo que se refiere a la cantidad total de complicaciones reportadas las mayores frecuencias fueron en infecciones de piel y tejidos blandos $(30.9 \% ; n=34 / 110)$, neumonía $(12.7 \% ; n=14 / 110)$, septicemia $(8.2 \%$; $\mathrm{n}=9 / 110)$, trastorno de la coagulación $(7.3 \%$; $n=8 / 110)$, y encefalitis (4.5\%; $n=5 / 110)$ (Figura 1b). En pacientes hospitalizados las complicaciones más comunes fueron: infecciones de piel y tejidos blandos $(30.9 \% ; n=34 / 110)$ seguidas por otras complicaciones $(23.4 \% ; n=26 / 110)$ y neumonía (12.7\%; 14/110) (Figura 1c).

\section{Recursos de atención médica asociados con la varicela}

En el Cuadro 2 se resumen los recursos de atención médica asociados con la varicela. Los números promedio [IC95\%] de visitas ambulatorias fueron de $1.5[1.2-1.8]$ y de 1.6 [1.3-1.9] entre los pacientes ambulatorios y hospitalizados, respectivamente. La mayoría de los pacientes ambulatorios se atendió en consultorios médicos $(72.0 \%$; visitas en promedio [IC95\%] = 1.3 [1.0-1.7]), servicios de urgencias (34.7\%; 1.0 [0.7-1.4]), y clínicas ambulatorias de hospitales (16.0\%; 1.0 [0.5-1.7]). Por el contrario, $92.2 \%$ de los pacientes hospitalizados visitaron el servicio de urgencias (promedio [IC95\%] = $1.1[0.8-1.3]), 32.5 \%$ consulta externa (1.3 [0.9-1.8]), y $6.5 \%(1.0$ [0.4-2.2]) clínicas ambulatorias de hospitales. La duración promedio [IC95\%] de hospitalización fue de 6.7 [5.4-8.3] días.

En lo que respecta a prescripción de medicamentos, una proporción mayor de pacientes ambulatorios se trató con fármacos de venta libre versus los hospitalizados (80 vs $71.4 \%$, respectivamente), aunque una proporción menor recibió una receta para su cuadro de varicela (80 vs $94.8 \%$ ). En pacientes tratados con medicamentos de venta libre y prescritos, las tasas promedio de 


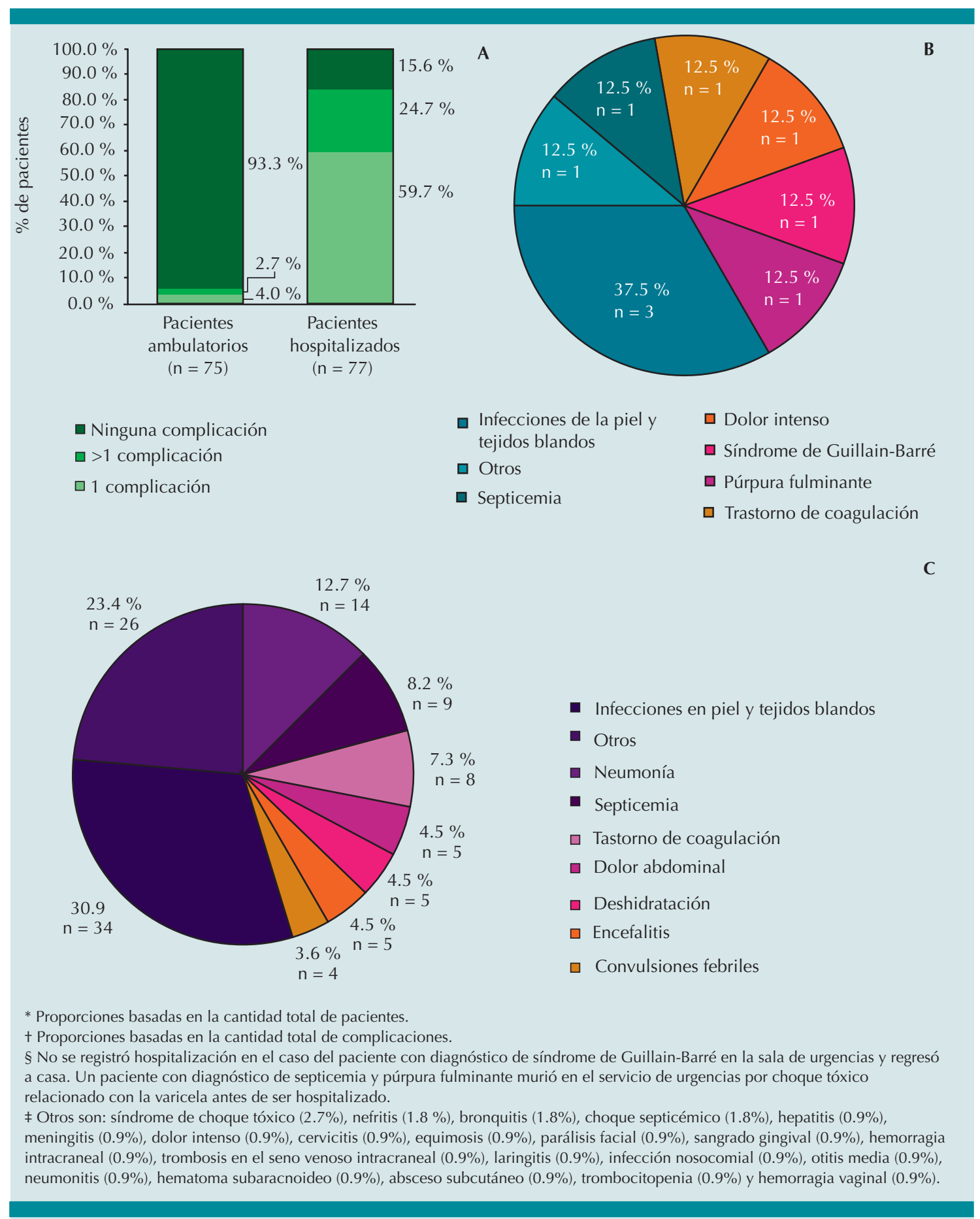

Figura 1. Complicaciones asociadas con la varicela. A) Porcentaje de pacientes con complicaciones, B) Compli-

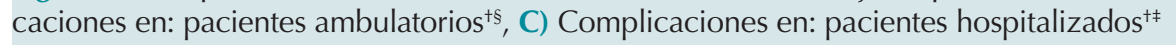


Cuadro 2. Uso de recursos de atención médica asociados con la varicela

\begin{tabular}{|c|c|c|c|c|}
\hline \multirow[t]{2}{*}{ Tipo } & \multicolumn{2}{|c|}{ Pacientes ambulatorios $(n=75)$} & \multicolumn{2}{|c|}{ Pacientes hospitalizados $(\mathbf{n}=77)$} \\
\hline & $\%$ de pacientes & Promedio [IC95 \% $]^{\dagger}$ & $\%$ de pacientes & Promedio [IC95\% $]^{\dagger}$ \\
\hline Visitas al consultorio médico & 72.0 & $1.3[1.0-1.7]$ & 32.5 & $1.3[0.9-1.8]$ \\
\hline Visitas a la sala de Urgencias & 34.7 & $1.0[0.7-1.4]$ & 94.7 & $1.1[0.8-1.3]$ \\
\hline $\begin{array}{l}\text { Visitas a clínicas ambulato- } \\
\text { rias de hospitales* }\end{array}$ & 16.0 & $1.0[0.5-1.7]$ & 6.5 & $1.0[0.4-2.2]$ \\
\hline Visitas ambulatorias totales ${ }^{\ddagger}$ & 100 & $1.5[1.2-1.8]$ & 96.1 & $1.6[1.3-1.9]$ \\
\hline Hospitalización ${ }^{\S}$ & $\mathrm{N} / \mathrm{C}$ & $\mathrm{N} / \mathrm{C}$ & 100 & $6.7[5.4-8.3]$ \\
\hline Estancia en la UCI & $\mathrm{N} / \mathrm{C}$ & $\mathrm{N} / \mathrm{C}$ & 19.5 & $5.4[3.7-7.8]$ \\
\hline Medicamentos recetados & 80.0 & $1.2[0.9-1.4]$ & 94.8 & $2.9[2.6-3.4]$ \\
\hline Medicamentos de venta libre & 80.0 & $1.7[1.4-2.0]$ & 71.4 & $4.0[3.5-4.6]$ \\
\hline Análisis y procedimientos & 5.3 & $5.7[3.8-8.7]$ & 87.0 & $5.0[4.5-5.6]$ \\
\hline $\begin{array}{l}\text { Consultas a profesionales de } \\
\text { la salud no médicos** }\end{array}$ & 12.0 & $1.0[0.5-1.8]$ & 61.0 & $8.2[7.4-9.1]$ \\
\hline
\end{tabular}

$\mathrm{N} / \mathrm{C}=$ no corresponde.

+ Se refiere al promedio de ocasiones en que se usa cada recurso de atención médica entre los usuarios; en los casos de hospitalización y estancia en la $\mathrm{UCl}$, se refiere a la duración en días.

* Definidas como visitas a la clínica o departamento ambulatorio de un hospital para consulta médica

* Suma de visitas a consultorios médicos, urgencias y clínicas ambulatorias de hospitales.

$\S$ La hospitalización no incluye estancia en la UCl.

** Definidas como consultas a fisioterapéutas, psicólogos, médicos especialistas, trabajadores sociales u "otros" profesionales de la salud.

uso [IC95\%] en los hospitalizados tratados con medicamentos de venta libre (pacientes ambulatorios: 1.7 [1.4-2.0]; pacientes hospitalizados: 4.0 [3.5-4.6] medicamentos por usuario) y con fármacos recetados (pacientes ambulatorios: 1.2 [0.9-1.4]; pacientes hospitalizados: 2.9 [2.6-3.4]) fueron más altas. A la mayoría $(87.0 \%)$ de los pacientes hospitalizados se les realizaron análisis o procedimientos (promedio [IC95\%] = $5.0[4.5$ 5.6]), y $61.0 \%$ requirió consultas a profesionales de la salud no médicos (8.2 [7.4-9.1]).

\section{Costos}

En el Cuadro 3a se encuentran los costos unitarios de los principales parámetros de recursos de atención médica. Esos costos se usaron para calcular los costos directos por caso de varicela en pacientes ambulatorios y hospitalizados por tipo de recurso utilizado (Cuadro $3 \mathbf{b}$ ). En general, los gastos de la atención médica directa fueron más altos en los pacientes hospitalizados que en los ambulatorios (promedio [IC95\%]; pacientes hospitalizados vs ambulatorios: 5,610.99 [4,113.63-7,108.35] vs 198.31 [171.64-224.99] dólares). Los costos directos de los pacientes ambulatorios se debieron, principalmente, a visitas a consultorios médicos (promedio [IC95 \%]: 83.43 [65.59-101.26] dólares), seguidos de las visitas a urgencias (45.74 [31.19- 60.28] dólares). La hospitalización representó la mayor parte de los gastos de los pacientes hospitalizados (promedio [IC95\%]: 2,845.11 [2,291.93-3,398.28] dólares). Los cos- 
Cuadro 3a. Costo promedio de los recursos de atención médica

\begin{tabular}{l|c|c|}
\hline Recurso de atención médica & $\begin{array}{c}\text { Tipo de } \\
\text { moneda }\end{array}$ & $\begin{array}{c}\text { Costo } \\
\text { promedio }\end{array}$ \\
\hline $\begin{array}{l}\text { Visitas al consultorio } \\
\text { médico }\end{array}$ & MXN & 1,704 \\
\hline Visitas a urgencias & MXN & 87 \\
\hline $\begin{array}{l}\text { Visitas a clínicas ambulato- } \\
\text { rias de hospitales* }\end{array}$ & dólares & 132 \\
\hline Día de hospitalización & dólares & 674 \\
\hline $\begin{array}{l}\text { Día de estancia en cuida- } \\
\text { dos intensivos }\end{array}$ & MXN & 7,256 \\
\hline & dólares & 370 \\
\hline
\end{tabular}

$\mathrm{MXN}=$ pesos mexicanos; dólares = dólares estadounidenses . $1 \mathrm{MXN}=0.051$ dólares. ${ }^{27}$

${ }^{+}$La fuente de costos es el Instituto Mexicano del Seguro Social. ${ }^{13}$

* Definidas como visitas a la clínica o departamento ambulatorio de un hospital para consulta médica

* El costo de hospitalización no incluye el de estancia en cuidados intensivos

tos indirectos fueron más elevados en el grupo de pacientes hospitalizados que en los ambulatorios (promedio [IC95\%]. De los hospitalizados vs ambulatorios: 175.21 [136.46-213.95] vs 42.33 [N/C] dólares. En general, el costo promedio [IC95\%] fue de 5,786.20 [4,251.93-7,320.47] dólares y 240.65 [213.97-267.33] dólares, por caso de varicela entre pacientes hospitalizados y ambulatorios, respectivamente.

En el Cuadro 4a está el costo anual estimado atribuible a la varicela en pacientes pediátricos de México, según las estimaciones por caso de varicela que se encuentran en el Cuadro 3b, y la incidencia promedio de varicela de 2011-2016.

Después de desglosar por parámetro de costo directo, las hospitalizaciones representaron $32.17 \%$ de los gastos directos, seguidas de las

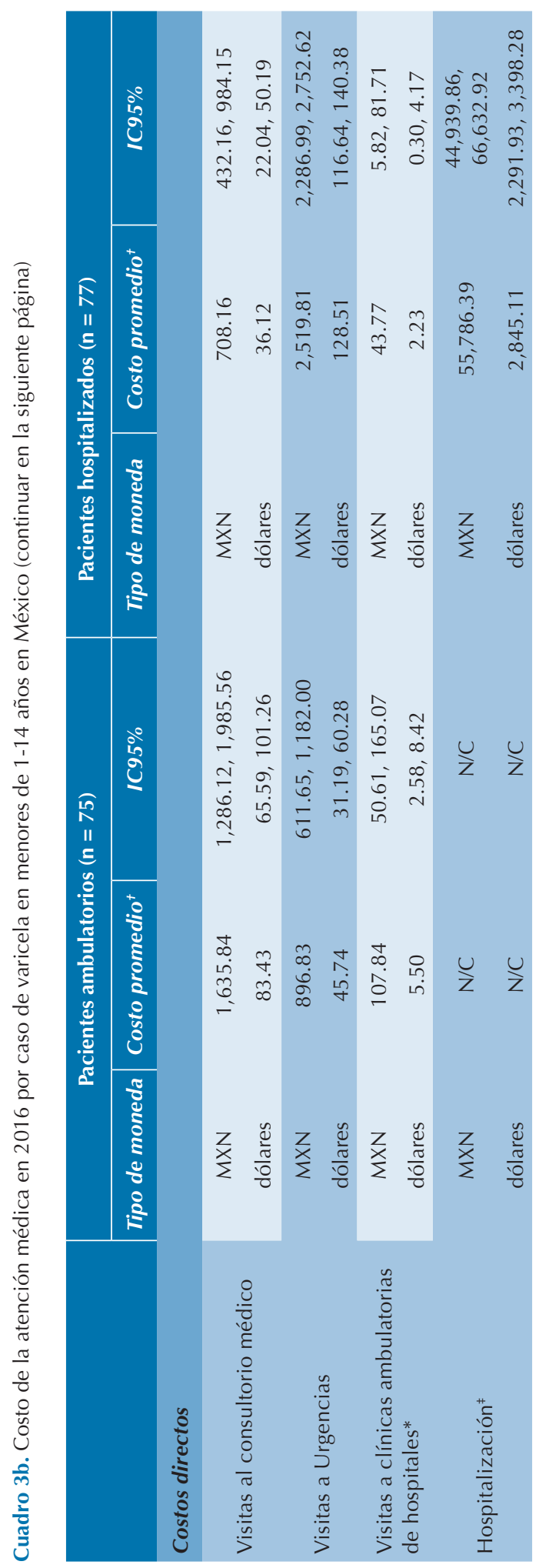




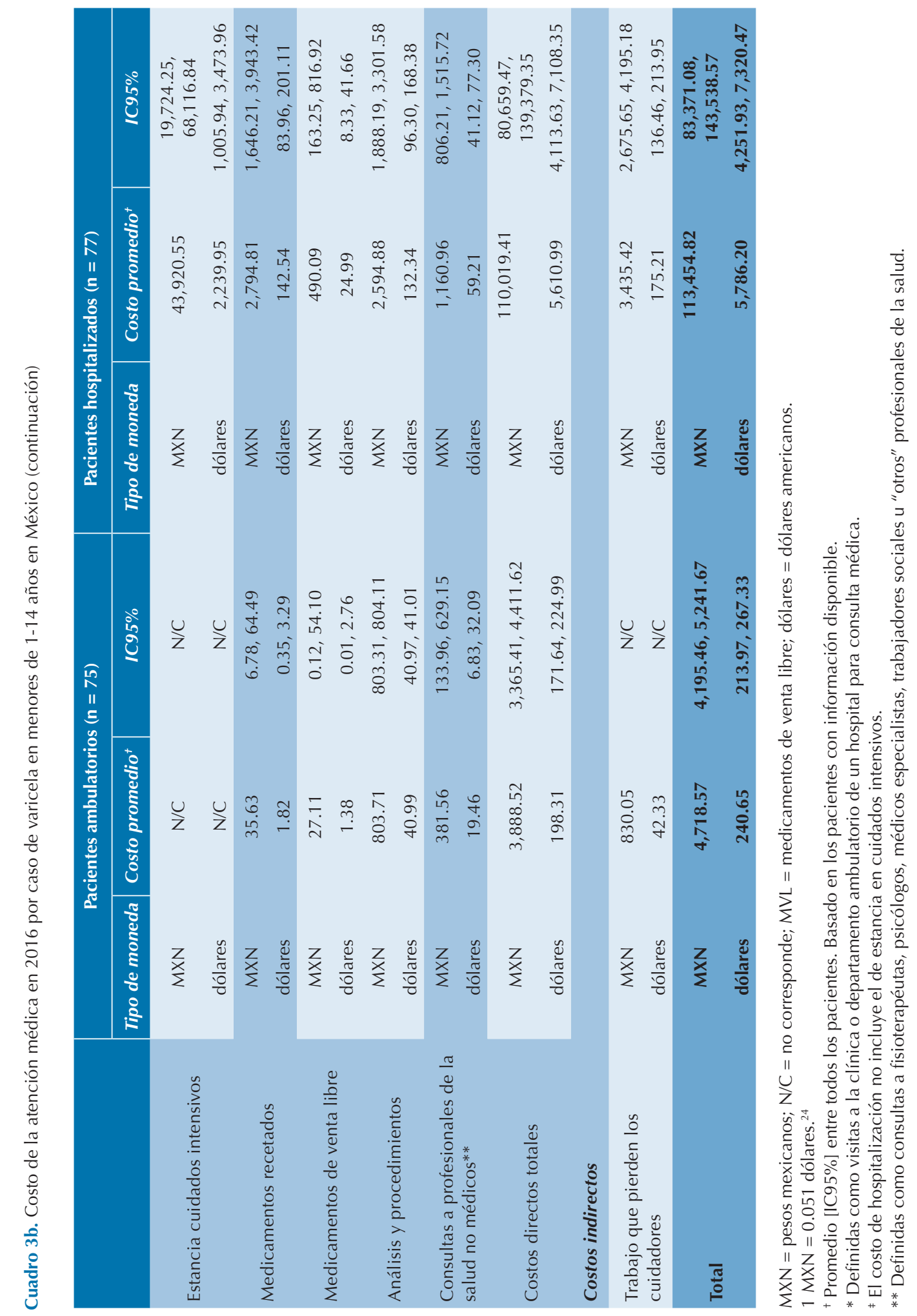


Cuadro 4a. Costos anuales generales, directos e indirectos, estimados (en millones) de menores de 1-14 años con varicela en México, 2011-2016

\begin{tabular}{|c|c|c|c|}
\hline & Tipo de moneda & Promedio (2011-2016) & $\%$ de los costos directos totales \\
\hline \multicolumn{4}{|l|}{ Costos directos } \\
\hline \multirow{2}{*}{ Visitas al consultorio médico } & $M X N$ & 348.15 & \multirow[t]{2}{*}{15.79} \\
\hline & dólares & 17.76 & \\
\hline \multirow{2}{*}{ Ingresos a Urgencias } & $M X N$ & 217.98 & \multirow[t]{2}{*}{9.88} \\
\hline & dólares & 11.12 & \\
\hline \multirow{2}{*}{ Visitas a clínicas ambulatorias* } & $M X N$ & 22.91 & \multirow[t]{2}{*}{1.04} \\
\hline & dólares & 1.17 & \\
\hline \multirow{2}{*}{ Hospitalización } & $M X N$ & 709.51 & \multirow[t]{2}{*}{32.17} \\
\hline & dólares & 36.18 & \\
\hline \multirow{2}{*}{ Estancia en cuidados intensivos } & $M X N$ & 558.60 & \multirow[t]{2}{*}{25.33} \\
\hline & dólares & 28.49 & \\
\hline \multirow{2}{*}{ Medicamentos recetados } & $M X N$ & 42.93 & \multirow[t]{2}{*}{1.95} \\
\hline & dólares & 2.19 & \\
\hline \multirow{2}{*}{ Medicamentos de venta libre } & MXN & 11.85 & \multirow[t]{2}{*}{0.54} \\
\hline & dólares & 0.60 & \\
\hline \multirow{2}{*}{ Análisis y procedimientos } & MXN & 199.63 & \multirow[t]{2}{*}{9.05} \\
\hline & dólares & 10.18 & \\
\hline \multirow{2}{*}{$\begin{array}{l}\text { Consultas a profesionales de la salud } \\
\text { no médicos** }\end{array}$} & MXN & 93.87 & \multirow{2}{*}{4.26} \\
\hline & dólares & 4.79 & \\
\hline \multirow{2}{*}{ Costos directos totales } & $M X N$ & $2,205.45$ & \multirow{2}{*}{100.00} \\
\hline & dólares & 112.48 & \\
\hline \multicolumn{4}{|l|}{ Costos indirectos } \\
\hline \multirow{2}{*}{$\begin{array}{l}\text { Días de trabajo que pierden los } \\
\text { cuidadores }\end{array}$} & $\mathrm{MXN}$ & 215.78 & \multirow{2}{*}{-} \\
\hline & dólares & 11.00 & \\
\hline \multirow{2}{*}{ Total (costos directos e indirectos) } & MXN & $2,421.23$ & \multirow[b]{2}{*}{-} \\
\hline & dólares & 123.48 & \\
\hline
\end{tabular}

$\mathrm{MXN}=$ pesos mexicanos; $\mathrm{N} / \mathrm{C}=$ no corresponde; $\mathrm{MVL}=$ medicamentos de venta libre; dólares = dólares americanos . $1 \mathrm{MXN}=0.051$ dólares $^{25}$

+ Las estimaciones del costo por caso de varicela aplicadas, se derivan del Cuadro 3 b y se aplican al número promedio de casos de varicela en el escenario básico reportados al SUIVE en el período 2011-2016, lo que representa la proporción estimada de solicitantes de atención médica que no se captura en el programa de vigilancia. ${ }^{2}$

* Definidas como visitas a la clínica o departamento ambulatorio de un hospital para consulta médica.

** Definidas como consultas a fisioterapéutas, psicólogos, médicos especialistas, trabajadores sociales u "otros" profesionales de la salud.

estancias en cuidados intensivos (25.33\%), las visitas a consultorios médicos (15.79\%) y los ingresos a urgencias (9.88\%).
De acuerdo con la incidencia anual promedio de varicela por caso base, el costo anual total debido a ésta se estimó en 123.48 millones de 
dólares (costos directos: 112.48 millones de dólares; costos indirectos: 11.00 millones de dólares).

Conforme al análisis de sensibilidad, que se resume en el Cuadro $\mathbf{4 b}$, el costo total anual estimado relacionado con la varicela fluctuó de 102.16 a 154.35 millones de dólares, con 93.05-140.60 millones de dólares por costos directos y 9.10-13.76 millones de dólares por costos indirectos.

Cuadro 4b. Costos anuales generales, directos e indirectos, estimados y totales (en millones) de los menores de 1-14 años con varicela en México, 2011-2016; análisis de sensibilidad

\begin{tabular}{|c|c|c|}
\hline & $\begin{array}{l}\text { Tipo de } \\
\text { moneda }\end{array}$ & $\begin{array}{l}\text { Promedio } \\
(2011-2016)\end{array}$ \\
\hline \multicolumn{3}{|l|}{ Límite inferior $^{+}$} \\
\hline \multirow{2}{*}{ Costos directos totales } & MXN & $1,824.61$ \\
\hline & dólares & 93.05 \\
\hline \multirow{2}{*}{$\begin{array}{l}\text { Costos indirectos: trabajo } \\
\text { que pierden los cuidadores }\end{array}$} & $M X N$ & 178.52 \\
\hline & dólares & 9.10 \\
\hline \multirow{2}{*}{ Total } & MXN & $2,003.13$ \\
\hline & dólares & 102.16 \\
\hline \multicolumn{3}{|l|}{ Límite superior ${ }^{*}$} \\
\hline \multirow{2}{*}{ Costos directos totales } & $M X N$ & $2,756.81$ \\
\hline & dólares & 140.60 \\
\hline \multirow{2}{*}{$\begin{array}{l}\text { Costos indirectos: trabajo } \\
\text { que pierden los cuidadores }\end{array}$} & MXN & 269.73 \\
\hline & dólares & 13.76 \\
\hline \multirow{2}{*}{ Total } & MXN & $3,026.53$ \\
\hline & dólares & 154.35 \\
\hline
\end{tabular}

MXN = pesos mexicanos; dólares = dólares americanos $1 \mathrm{MXN}=0.051$ dólares $^{27}$

+ Las estimaciones de los costos por caso de varicela aplicadas se derivan del Cuadro 3b, y se aplican a la cifra promedio bruta de casos de varicela reportados al SUIVE durante 2011-2016.18-23.

₹ Las estimaciones del costo por caso de varicela aplicadas se derivan de el Cuadro 3b, y se aplican al escenario básico inflado en $25 \%$ para reflejar la proporción estimada de pacientes del sector público que utilizan servicios privados (límite superior). ${ }^{25}$

\section{DISCUSIÓN}

Los hallazgos de este estudio retrospectivo destacan la considerable carga de atención clínica y médica asociada con las infecciones de varicela pediátrica primaria en México, en pacientes ambulatorios y hospitalizados. En el entorno social, la atención médica de pacientes pediátricos con varicela representa una considerable carga económica para el sistema de salud pública y los cuidadores. Alrededor de $9 \%$ de los costos generales asociados con la varicela es atribuible a gastos indirectos por pérdida de trabajo. En general, los costos totales anuales estimados debidos a la varicela fueron altos, pues ascienden, aproximadamente, a 123 millones de dólares por año en el caso del escenario básico.

En lo que concierne a complicaciones, la tasa más alta en pacientes hospitalizados (pacientes hospitalizados vs ambulatorios: 84.4vs $6.7 \%$ ) concuerda con los hallazgos publicados. En los estudios italianos, alemanes y suizos se reportaron tasas de complicaciones relacionadas con la varicela de $3.5,5.9$ y $12.0 \%$, respectivamente, en pacientes ambulatorios, ${ }^{28,29,30}$ mientras que las tasas de complicaciones en los hospitalizados fluctuaron entre 50 y $72 \%$ en América Latina. ${ }^{31,32}$ La tasa más alta de complicaciones en pacientes hospitalizados y ambulatorios de este estudio, en comparación con otros países de América Latina, podría atribuirse a la mayor gravedad de la enfermedad en México, a diferencias atribuibles al acceso a centros de atención médica, a la exactitud de la recopilación de datos, y a diferencias en la frecuencia de reporte de la enfermedad.

La tasa de complicación en México reportada en este estudio coincide con las estimaciones en América Latina. En los estudios efectuados por Anjos y su grupo, ${ }^{33}$ Sáez-Llorens y colaboradores ${ }^{31}$ y Abarca y sus colegas ${ }^{32}$ que evaluaron las complicaciones de la varicela en pacientes hospitalizados, las infecciones cutáneas fueron la 
complicación predominante de la varicela, comparable con los hallazgos actuales. Además, en coincidencia con los hallazgos de este estudio, Sáez-Llorens y colaboradores ${ }^{31}$ y Abarca y su grupo $^{32}$ reportaron tasas bajas de eventos neurológicos en pacientes pediátricos con varicela en hospitales de Panamá y Chile, respectivamente.

Si bien la vacuna contra la varicela se administra en México a un subconjunto de pacientes de alto riesgo del sector de salud pública, ${ }^{34}$ muchos países de América Latina han implementado programas de inmunización contra la varicela que logran tasas de cobertura de más de $90 \%$, lo que ha reducido el uso de recursos de atención médica. ${ }^{35,36,37}$ En Uruguay, la introducción de la vacuna contra la varicela al programa de inmunización tuvo lugar en 1999, con vacunación de rutina de los lactantes a los 12 meses de edad; en 2005 se reportó una clara disminución de las hospitalizaciones pediátricas relacionadas con la varicela $(81 \%)$ y de las consultas ambulatorias $(87 \%)$ al Centro Hospitalario Pereira Rossell de Montevideo. ${ }^{37}$ Se observaron mejorías similares en Costa Rica desde el comienzo de la vacunación obligatoria contra la varicela en septiembre de 2007: se reportaron declinaciones de 73.8 y $98.1 \%$ en la incidencia de varicela y hospitalizaciones en la población general, mientras que las reducciones respectivas fueron de 79.1 y $87 \%$ entre los menores de menos de 5 años de edad, respectivamente. ${ }^{36}$ Además, en un reciente análisis de rentabilidad mexicano se informó que el reemplazo de la vacuna triple (sarampión, parotiditis y rubéola, o SPR) por la vacuna SPR-varicela (SPRV) reduciría en cerca de $90 \%$ la incidencia de la varicela en México, y permitiría ahorrar, aproximadamente, $331 \mathrm{mi}$ Ilones de dólares en costos de atención médica durante 80 años, al tiempo que causaría tasas de complicaciones y mortalidad relacionadas con la varicela más bajas que la vacuna SPR actual. ${ }^{38}$

Los hallazgos actuales deben interpretarse teniendo en cuenta las limitaciones del estudio.
Si bien el SUIVE es un sistema de vigilancia epidemiológica sólido, las omisiones de reporte son frecuentes, sobre todo en lo que respecta a la varicela porque en ese caso el SUIVE no impone el reporte obligatorio. ${ }^{39}$ Por lo tanto, el número de casos utilizado en el escenario básico, si bien inflado para compensar los centros de atención médica que no envían reportes periódicos al SUIVE, podría, no obstante, representar una subestimación de la verdadera incidencia de la varicela. Esto, combinado con un diseño de estudio retrospectivo en el que la captura del uso de recursos de atención médica relacionado con la varicela se limitó a la información transcrita en los expedientes clínicos de los pacientes, pudo causar una subestimación de los costos directos por paciente y de los costos sociales totales. Por el contrario, puesto que quienes buscan atención médica podrían representar un subconjunto de pacientes con enfermedad más grave, es posible que se haya introducido un sesgo de selección cuyo resultado es una sobrestimación de la carga asociada con la varicela, aunque la extrapolación al costo nacional anual tuvo por objeto disminuir tal sesgo. Además, la generalización de los hallazgos pudiera verse limitada debido al escaso tamaño de la muestra y al número de centros participantes. Esto es especialmente relevante con respecto a los costos totales anuales relacionados con la varicela que se mencionan en este documento y que se extrapolan a partir de los datos proporcionados por los 152 pacientes incluidos. Por lo tanto, estos datos de costos deben interpretarse con precaución porque representan solo una estimación preliminar de la carga económica de la varicela pediátrica en México.

El uso de datos extraídos de los expedientes clínicos implica la posibilidad de sesgos en la información: datos faltantes, poco uniformes o erróneos y la probabilidad de errores humanos durante la transcripción. También es necesario destacar que, debido a la naturaleza retrospectiva 
de este estudio, no fue posible estimar los gastos médicos por desembolso directo reportados por $39.5 \%$ de los mexicanos cubiertos por seguros médicos privados y públicos. ${ }^{2}$ Por lo tanto, sigue pendiente la evaluación de la repercusión social total de las infecciones de varicela en México.

\section{CONCLUSIÓN}

Este es el primer estudio, hasta donde sabemos, que ha evaluado la carga económica asociada con la infección primaria de varicela en México. A pesar de las limitaciones mencionadas estos resultados ilustran la carga sustancial asociada con la infección pediátrica por varicela en México, incluidas las altas tasas de complicaciones, uso de recursos en salud y la carga del cuidador, proporcionando mayor comprensión de la verdadera repercusión de la varicela pediátrica, como se observa en los entornos de atención primaria. Además, las estimaciones de los costos anuales informados en este documento, aunque preliminares, pueden, no obstante, usarse en la comparación de análisis económicos futuros que evalúan la varicela pediátrica en México.

Además de las estimaciones de rentabilidad de la implementación de la vacuna SPRV previamente reportadas, así como los hallazgos relacionados con el efecto positivo de los programas de vacunación universal contra la varicela en América Latina y el resto del mundo, los hallazgos que se comunican en este documento destacan la necesidad de implementar en México un programa de vacunación de rutina contra la varicela entre los menores de edad.

\section{Agradecimientos}

Angela Karellis y Melissa Stutz de JSS Medical Research, Montreal, QC, Canadá, colaboraron en la redacción médica. Christina Mantizoros, ex empleada de JSS Medical Research, Montreal, QC, Canadá, dio apoyo administrativo al estudio.

\section{Financiamiento}

Merck, Kenilworth, Nueva Jersey, EUA, proporcionó apoyo financiero para el estudio. Todos los resultados y conclusiones del estudio reflejan la opinión de los autores y son independientes de la fuente de financiamiento.

\section{Conflictos de interés}

CPBV, HM, AA y LJW son empleados de Merck Sharp \& Dohme, una subsidiaria de Merck, que podrían poseer acciones o mantener opciones de acciones en la Compañía. MVR se ha desempeñado como consultor pago de Sanofi Pasteur y Merck y ha recibido apoyo para investigación por parte de Merck. ER es un empleado de JSS Medical Research, que fue contratado por Merck para llevar a cabo el estudio y proporcionar apoyo para la redacción de este escrito.

APR se ha desempeñado como consultor o asesor científico pago de Abbvie, BMS, Janssen, Gilead, Merck Sharpe \& Dohme y Stendhal y ha recibido apoyo para investigación por parte de Janssen. JAVN se ha desempeñado como conferencista pago y también ha recibido apoyo para investigación por parte de Merck Sharpe \& Dohme, Pfizer, Glaxo y Sanofi Pasteur. MAMR, RRR, CJV, ESM y EGF declaran que no existe ningún conflicto de interés.

\section{REFERENCIAS}

1. Arvin AM. Varicella-zoster virus. Clin Microbiol Rev. 1996;9(3):361-81.

2. Varicella and herpes zoster vaccines: WHO position paper, 20 June 2014. Wkly Epidemiol Rec 2014;89:265-87. Disponible en: http://www.who.int/wer/2014/wer8925/ en/. Consultado el: 12 de agosto de 2015.

3. Manfredi R, Chiodo F, Titone L, Vierucci A, Catania S, Ghirardini G, et al. Chickenpox complications among immunocompetent hospitalized children in Italy. Acyclovir-Chickenpox Italian Study Group. Pediatr Med Chir. 1997;19(2):99-104. 
4. Ozdemir H, Candir MO, Karbuz A, Belet N, Tapisiz A, Ciftci $\mathrm{E}$, et al. Chickenpox complications, incidence and financial burden in previously healthy children and those with an underlying disease in Ankara in the pre-vaccination period. Turk J Pediatr. 2011;53(6):614-25.

5. Ziebold C, von Kries R, Lang R, Weigl J, Schmitt HJ. Severe complications of varicella in previously healthy children in Germany: a 1-year survey. Pediatrics. 2001;108(5):E79.

6. Vázquez $\mathrm{M}$, et al. Varicela y herpes zóster: retos para la salud pública. Salud Publica Mex 2017;59:650-656. https://doi. org/10.21149/7997.

7. Prevención, diagnóstico y tratamiento de la varicela en el paciente pediátrico en el primer nivel de atención. Guía de referencia rápida o Guía de Práctica Clínica, México, Secretaría de Salud. CENETEC 25/05/2015. Disponible en: http://www.cenetec.salud.gob.mx/descargas/gpc/CatalogoMaestro/129_GPC_VARICELA/Issste_128_08_grr_varicela. pdf. Consultado el: 21 de julio de 2017.

8. Calendario en práctica privada. Disponible en: http:// www.centrodevacunacion.com.mx/Esquema\%20de $\% 20$ vacunas\%200\%20a\%2010\%20privado\%202007.pdf. 2007. Consultado el: 21 de julio de 2017.

9. Cartilla Nacional de Vacunación 2013. Disponible en: http:// www.censia.salud.gob.mx/contenidos/vacunas/esquemavacunas.html. 2013. Consultado el: 21 de julio de 2017.

10. Esquema de vacunación actual. Secretaría de Salud. Disponible en: http://www.censia.salud.gob.mx/contenidos/ vacunas/esquemavacunas.html. 2015. Consultado el: 21 de julio de 2017.

11. Vergara-Castañeda A, Escobar-Gutierrez A, Ruiz-Tovar K, Sotelo J, Ordonez G, Cruz-Rivera MY, et al. Epidemiology of varicella in Mexico. J Clin Virol. 2012;55(1):51-7.

12. Meszner Z, Molnar Z, Rampakakis E, Yang HK, Kuter BJ, Wolfson LJ. Economic burden of varicella in children 1-12 Years of age in Hungary, 2011-2015. BMC Infect Dis. 2017;17(1):495.

13. Diario Oficial de la Federación. Costos Unitarios por Nivel de Atención Médica, 2017. Disponible en: http://www.dof.gob. $\mathrm{mx} /$ nota_detalle.php?codigo=5476988\&fecha=21/03/2017. Consultado el: 21 de julio de 2017.

14. Instituto Mexicano del Seguro Social (IMSS). Compras. Disponible en: http://compras.imss.gob.mx/. Consultado el: 21 de julio de 2017.

15. Remuneraciones. Salario diario asociado a asegurados trabajadores en el Instituto Mexicano del Seguro Social. Disponible en: http://www.inegi.org.mx/sistemas/bie/cuadrosestadisticos/GeneraCuadro. aspx?s=est \&nc=583\&c=29478. Última modificación: 21 de julio de 2017. Consultado el: 28 de julio de 2017.

16. Ginsberg GM, Somekh E. Cost containment analysis of childhood vaccination against varicella in Israel. J Infect. 2004;48(2):119-33.

17. Law B, Fitzsimon C, Ford-Jones L, MacDonald N, Dery P, Vaudry W, et al. Cost of chickenpox in Canada: part I. Cost of uncomplicated cases. Pediatrics. 1999;104(1 Pt 1):1-6.
18. SUIVE/DGE/Secretaría de Salud/Estados Unidos Mexicanos. Dirección General de Epidemiología Anuario de Morbilidad 1984 -2016. Disponible en: http://www. epidemiologia.salud.gob.mx/anuario/html/anuarios.html

19. SUIVE/DGE/Secretaría de Salud/Estados Unidos Mexicanos. Casos nuevos de Varicela (B01) por grupos de edad. Estados Unidos Mexicanos 2012. Población General. Disponible en: http://www.epidemiologia.salud.gob.mx/ anuario/html/anuarios.html

20. SUIVE/DGE/Secretaría de Salud/Estados Unidos Mexicanos. Casos nuevos de Varicela (B01) por grupos de edad. Estados Unidos Mexicanos 2013. Población General. Disponible en: http://www.epidemiologia.salud.gob.mx/ anuario/html/anuarios.html

21. SUIVE/DGE/Secretaría de Salud/Estados Unidos Mexicanos. Casos nuevos de Varicela (B01) por grupos de edad. Estados Unidos Mexicanos 2014. Población General. Disponible en: http://www.epidemiologia.salud.gob.mx/ anuario/html/anuarios.html

22. SUIVE/DGE/Secretaría de Salud/Estados Unidos Mexicanos. Casos nuevos de Varicela (B01) por grupos de edad. Estados Unidos Mexicanos 2015. Población General. Disponible en: http://www.epidemiologia.salud.gob.mx/ anuario/html/anuarios.html

23. SUIVE/DGE/Secretaría de Salud/Estados Unidos Mexicanos. Casos nuevos de Varicela (B01) por grupos de edad. Estados Unidos Mexicanos 2016. Población General. Disponible en: http://www.epidemiologia.salud.gob.mx/ anuario/html/anuarios.html

24. Tapia-Conyer R, Kuri-Morales P, Gonzalez-Urban L, Sarti E. Evaluation and reform of Mexican National Epidemiological Surveillance System. Am J Public Health. 2001;91(11):175860.

25. Gertrudiz N. e-Health: the case of Mexico. Latin Am J Telehealth, Belo Horizonte. 2010; 2 (2): 127-167.

26. Bardach A, Cafferata ML, Klein K, Cormick G, Gibbons L, Ruvinsky S. Incidence and Use of Resources for Chickenpox and Herpes Zoster in Latin America and the Caribbean-A Systematic Review and Meta-analysis. Pediatr Infect Dis J. 2012;31(12):1263-8

27. Organisation for Economic Co-Operation and Development. Monthly Monetary and Financial Statistics (MEI): Exchange rates (dólares monthly averages). Disponible en: http://stats.oecd.org/index.aspx?queryid=169. Consultado el 28 de julio de 2017.

28. Fornaro P, Gandini F, Marin M, Pedrazzi C, Piccoli P, Tognetti $D$, et al. Epidemiology and cost analysis of varicella in Italy: results of a sentinel study in the pediatric practice. Italian Sentinel Group on Pediatric Infectious Diseases. Pediatr Infect Dis J. 1999;18(5):414-9.

29. Iseli A, Aebi C, Banz K, Brunner M, Schmutz AM, Heininger $U$. Prospective surveillance of varicella-zoster virus infections in an out-patient setting in Switzerland. Hum Vaccin. 2009;5(12):843-6.

30. Wagenpfeil S, Neiss A, Banz K, Wutzler P. Empirical data on the varicella situation in Germany for vaccination decisions. Clin Microbiol Infect. 2004;10(5):425-30. 
31. Sáez-Llorens X dSO, de Morós D, del Pilar Rubio M. Complicaciones y costos asociados a la varicela en niños inmunocompetentes. Rev Panam Salud Publica.12(2).

32. Abarca K HT, Potin M, Perret C, Zamorano J, González C, Vial P. [Complications in children with varicella in 4 hospitals in Santiago, Chile: clinical spectrum and estimation of direct costs]. Article in Spanish. Rev Med Chil 129(4):397-404.

33. Anjos KS EFM, Ceu Arruda M, da Silva Ramos K, Regazzi Magalhars AP. [Caracterização epidemiológica dos casos de varicela em pacientes internados em um hospital universitário da cidade do Recife]. Article in Portugese. Rev bras epidemiol São Paulo vol.12 (no.4).

34. World Health Organization. WHO vaccine-preventable diseases: monitoring system. 2017 global summary. http:// apps.who.int/immunization_monitoring/globalsummary/ schedules?sc\%5Br\%5D\%5B\%5D=AMRO\&sc\%5Bd\%5D= \&sc\%5Bv\%5D\%5B\%5D=VARICELLA\&sc\%5BOK\%5D=OK. Consultado el: 18 de julio de 2017.

35. WHO vaccine-preventable diseases: monitoring system. 2016 global summary. Disponible en http://apps.who. int/immunization_monitoring/globalsummary/schedules.
36. Avila-Aguero ML, Ulloa-Gutierrez R, Camacho-Badilla $\mathrm{K}$, Soriano-Fallas A, Arroba-Tijerino R, Morice-Trejos A. Varicella prevention in Costa Rica: impact of a one-dose schedule universal vaccination. Expert Rev Vaccines. 2017;16(3):229-34.

37. Quian J, Ruttimann R, Romero C, Dall'Orso P, Cerisola A, Breuer $T$, et al. Impact of universal varicella vaccination on 1-year-olds in Uruguay: 1997-2005. Arch Dis Child. 2008;93(10):845-50.

38. Ouwens M, Macias M, Mascareñas De Los Santos AH, Gomez JA, Sauboin C, Carreño Manjarrez R. Replacing MMR by MMRV in Mexico: Assessment of Cost-Effectiveness Based on a Dynamic Transmission Model. Abstract presented at the ISPOR 3rd Latin America Conference, Mexico City, Mexico. September 2011.

39. Prevención, Diagnóstico y Tratamiento de la Varicela Infantil en el primer nivel de atención. México: Secretaria de Salud; 2008. Disponible en: http://sgm.issste.gob. $\mathrm{mx} /$ medica/medicadocumentacion/guiasautorizadas/ Dermatolog\%C3\%ACa/ISSSTE-129_08_VARICELA_INFANTIL/ISSSTE-129-08-GPC\%20EYR\%20VARICELA\%202011.pdf

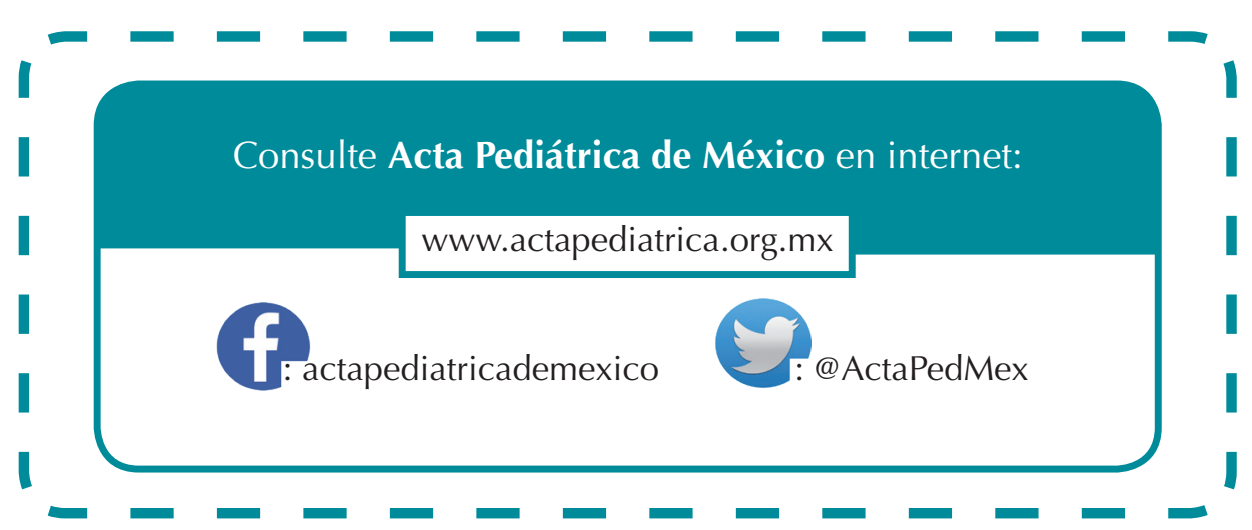

